\title{
LIOUVILLE'S THEOREM FOR PARABOLIC EQUATIONS OF THE SECOND ORDER WITH CONSTANT COEFFICIENTS ${ }^{1}$
}

\author{
AVNER FRIEDMAN
}

1. We shall consider a generalization of Liouville's Theorem for functions which are solutions of the parabolic equation

$$
\frac{\partial u(x, t)}{\partial t}=\sum_{i, j=1}^{m} a_{i j} \frac{\partial^{2} u(x, t)}{\partial x_{i} \partial x_{j}}+\sum_{i=1}^{m} b_{i} \frac{\partial u(x, t)}{\partial x_{i}}+c u(x, t) .
$$

The coefficients $a_{i j}, b_{i}$ and $c$ are real constants, $\left(a_{i j}\right)$ is a positive matrix and the solutions $u(x, t)$ are defined and nonnegative in the half space $-\infty<t \leqq 0$, denoted by $D$. We use the notation $x$ $=\left(x_{1}, \cdots, x_{m}\right),|x|=\left(\sum x_{i}^{2}\right)^{1 / 2}$.

It will be shown later on, that the nontrivial solutions of (1) are positive in the interior of $D$ and that

$$
\lim _{t \rightarrow-\infty} \frac{\log u(0, t)}{t} \text { exists. }
$$

The growth properties of these solutions will be studied in cones $D_{\alpha}$ : $|x| \leqq \alpha|t|, t<0$ with axis $x=0$ and opening $2 \alpha$. The natural generalization of Liouville's Theorem (see, for instance, [1]) is equivalent to the statement that the nonconstant solutions of (1) cannot be bounded in $D$, which may be considered as a cone $D_{\alpha}$ with $\alpha=\infty$. We can now state the main result of this paper.

THEOREM 1. Let $u(x, t)$ be a nontrivial nonnegative solution of (1) in the half space D. Denote

$$
\lim _{t \rightarrow-\infty} \frac{\log u(0, t)}{t}=c+\gamma .
$$

If $c+\gamma \geqq 0$ and if $\gamma>0$, then $u(x, t)$ is unbounded in $D_{\alpha}$ for all

$$
\alpha>(c+\gamma)\left(\left(\gamma+\frac{1}{4} b^{2}\right)^{1 / 2}-\frac{1}{2} b\right)^{-1},
$$

where $b=\left(\sum b_{i}^{2}\right)^{1 / 2}$.

The assumption $c+\gamma \geqq 0$ excludes the trivial case $c+\gamma<0$, in

Received by the editors August 3, 1957 and, in revised form, September 23, 1957.

${ }_{1}$ Paper written under contract with Office of Naval Research N58304. 
which $u(0, t)$ is unbounded. The assumption $\gamma>0$ is sharp in the sense that if $\gamma=0, u$ may be bounded in $D$. As an example, take $u(x, t)=e^{c t}$ where $c>0 ; u(x, t)$ satisfies $\partial u / \partial t=\Delta u+c u$, but it is bounded in $D$. Note finally, that the assumption $\gamma>0$ excludes constant $(\neq 0)$ solutions. The proof of Theorem 1 is given in the next two sections.

2. In this section we shall consider some growth properties of a special class of nontrivial solutions of the heat equation

$$
\frac{\partial w(x, t)}{\partial t}=\Delta w(x, t)
$$

in the half space $D$. These solutions are assumed to be nonnegative and symmetric in $x$ with respect to the axis $x=0$, i.e., $w(x, t)=u(|x|, t)$. Separating variables in (5), we easily find that

$$
e^{\xi^{2} t} K_{m}(|x| \xi)=e^{\xi^{2} t} \sum_{k=0}^{\infty} \frac{(\xi|x|)^{2 k}}{4^{k} k ! \Gamma(k+m / 2)}
$$

is a nonnegative symmetric solution of (5), and by superposition, we obtain solutions of the form

$$
\begin{aligned}
w(x, t)=u(|x|, t)= & \int_{0}^{\infty} e^{\xi^{2} t} K_{m}(|x| \xi) d \phi(\xi) \\
& \left(\phi(\xi) \nearrow ; 0<\int_{0}^{\infty} d \phi(\xi)<\infty\right) .
\end{aligned}
$$

Hirschman [2] has proved that every nonnegative symmetric solution of (5) can be written in the form (7). The representation (7) will play an essential role in the following.

We can define

$$
h(\alpha)=\limsup _{t \rightarrow-\infty} \frac{\log u(\alpha|t|, t)}{t},
$$

since, as follows from (7), the nonnegative symmetric solutions of (5) in $D$ are positive in the interior of $D$. Let $\alpha_{0}$ denote the distance from the origin to the support of $d \phi(\xi)$.

Lemma 1. If $0 \leqq \alpha \leqq \alpha_{0}$; then $h(\alpha)=\alpha_{0}\left(\alpha_{0}-\alpha\right)$.

Proof. Consider first the function

$$
v(\alpha|t|, t)=\int_{0}^{\infty} e^{(\xi-\alpha) \xi t} d \phi(\xi)
$$


From the definition of $\alpha_{0}$ it follows that for every $\epsilon>0$

$$
v(\alpha|t|, t)=\left(\int_{\alpha_{0}}^{\alpha_{0}+\epsilon} e^{\xi(\xi-\alpha) t} d \phi(\xi)\right)(1+o(1)) \quad(t \rightarrow-\infty) .
$$

Consequently,

$$
A_{1} e^{\left(\alpha_{0}+\epsilon\right)\left(\alpha_{0}+\epsilon-\alpha\right) t} \leqq v(\alpha|t|, t) \leqq A_{2} e^{\alpha_{0}\left(\alpha_{0}-\alpha\right) t} \quad\left(A_{1}>0, A_{2}>0\right),
$$

from which it follows that

$$
\limsup _{t \rightarrow-\infty} \frac{\log v(\alpha|t|, t)}{t}=\alpha_{0}\left(\alpha_{0}-\alpha\right) .
$$

Using the inequalities

$$
\begin{array}{r}
A^{\prime} K_{m}(\alpha|t| \xi) \leqq e^{\alpha \xi|t|} \leqq A K_{m}(\alpha(1+\epsilon)|t| \xi) \\
\left(A^{\prime}>0, A=A(\epsilon)>0\right),
\end{array}
$$

which follow by comparing the corresponding power series expansions, and using the definitions (8) and (9), we conclude from (11) that

$$
\alpha_{0}\left(\alpha_{0}-\alpha\right) \leqq h(\alpha), \quad h(\alpha(1+\epsilon)) \leqq \alpha_{0}\left(\alpha_{0}-\alpha\right) .
$$

Replacing $\alpha$ in the second inequality by $\alpha /(1+\epsilon)$ and taking $\epsilon \rightarrow 0$, we obtain $h(\alpha) \leqq \alpha_{0}\left(\alpha_{0}-\alpha\right)$, which together with the first inequality proves the lemma.

From the inequalities (10) it is clear that we have actually proved that $\lim _{t \rightarrow-\infty}(\log u(\alpha|t|, t)) / t$ exists and is equal to $\alpha_{0}\left(\alpha_{0}-\alpha\right)$. Taking in particular $\alpha=0$, we obtain

$$
\alpha_{0}^{2}=\lim _{t \rightarrow-\infty} \frac{\log u(0, t)}{t} .
$$

Lemma 2. If $\alpha_{0}<\alpha<\infty$, then $h(\alpha) \leqq \alpha_{0}\left(\alpha_{0}-\alpha\right)$.

Proof. As in the proof of the preceding lemma, we first consider the functon $v(\alpha|t|, t)$ defined by (9).

Since $d \phi(\xi) \not \equiv 0$ immediately to the right of $\xi=\alpha_{0}$, we get

$$
\int_{\alpha_{0}}^{\alpha} e^{\xi(\xi-\alpha) t} d \phi(\xi) \geqq A e^{\left(\alpha_{0}+\epsilon\right)\left(\alpha_{0}+\epsilon-\alpha\right) t} \quad(\epsilon>0, A=A(\epsilon)>0),
$$

from which it follows that

$$
\limsup _{t \rightarrow-\infty} \frac{\log v(\alpha|t|, t)}{t} \leqq\left(\alpha_{0}+\epsilon\right)\left(\alpha_{0}+\epsilon-\alpha\right) .
$$


Taking $\epsilon \rightarrow 0$ and proceeding as in the proof of Lemma 1, we finally get $h(\alpha) \leqq \alpha_{0}\left(\alpha_{0}-\alpha\right)$.

3. Let $z(x, t)$ be a nontrivial nonnegative solution of the heat equation in $D$. Define

$$
u(r, t)=\frac{1}{\omega_{m} r^{m-1}} \int_{|x|=r} z(x, t) d S_{x},
$$

where $\omega_{m}$ is the surface area of the $m$-dimensional unit sphere. $u(|x|, t)$ is also a solution of the heat equation [2] and, consequently, belongs to the class of functions considered in the preceding section. Denoting

$$
M_{t}^{2}(r)=\underset{|x|=r}{\operatorname{Max}} z(x, t),
$$

and applying Lemmas 1, 2 and (13), we obtain

LEMMA 3. For every nonnegative $\alpha$,

$$
\limsup _{t \rightarrow-\infty} \frac{\log M_{t}^{2}(\alpha|t|)}{t} \leqq \alpha_{0}\left(\alpha_{0}-\alpha\right)
$$

where $\alpha_{0}$ is given by

$$
\alpha_{0}^{2}=\lim _{t \rightarrow-\infty} \frac{\log z(0, t)}{t} .
$$

With the aid of Lemma 3 we proceed to prove Theorem 1. Let $T=\left(t_{i j}\right)$ be an orthogonal matrix such that the function $v(x, i)$ $=u(T x, t)$ satisfies the equation

$$
\frac{\partial v(x, t)}{\partial t}=\Delta v(x, t)+\sum_{i=1}^{m} d_{i} \frac{\partial v(x, t)}{\partial x_{i}}+c v(x, t) .
$$

Clearly, $d=\left(\sum d_{t}^{2}\right)^{1 / 2}=\left(\sum b_{i}^{2}\right)^{1 / 2}=b$. The function $w(x, t)=e^{\Sigma d_{i} x_{i} / 2} v(x, t)$ satisfies the equation

$$
\frac{\partial w(x, t)}{\partial t}=\Delta w(x, t)+\left(c-b^{2} / 4\right) w(x, t)
$$

and finally, the function $z(x, t)=e^{-\left(c-b^{2} / 4\right) t} w(x, t)$ is a nontrivial nonnegative solution of the heat equation. Since

$$
u(T x, t)=e^{\left(c-b^{2} / 4\right) t} e^{-\Sigma d_{i} x_{i} / 2} z(x, t),
$$

it follows that $u(x, t)$ is positive in the interior of $D$ and that 


$$
\lim _{t \rightarrow-\infty} \frac{\log u(0, t)}{t}=\left(c-\frac{1}{4} b^{2}\right)+\lim _{t \rightarrow-\infty} \frac{\log z(0, t)}{t} .
$$

From (17) we also deduce that

$$
\begin{aligned}
\limsup _{t \rightarrow-\infty} & \frac{\log M_{t}^{u}(\alpha|t|)}{t} \\
& \leqq\left(c-\frac{1}{4} b^{2}\right)+\frac{1}{2} b \alpha+\limsup _{t \rightarrow-\infty} \frac{\log M_{t}^{z}(\alpha|t|)}{t} .
\end{aligned}
$$

From (3) and (18) we have

$$
\lim _{t \rightarrow-\infty} \frac{\log z(0, t)}{t}=\gamma+\frac{1}{4} b^{2}
$$

and using Lemma 3 we obtain,

$$
\limsup _{t \rightarrow-\infty} \frac{\log M_{t}^{z}(\alpha|t|)}{t} \leqq \nu+b^{2} / 4-\left(\gamma+b^{2} / 4\right)^{1 / 2} \alpha .
$$

Substituting in (19) and taking $\alpha>(c+\gamma)\left(\left(\gamma+b^{2} / 4\right)^{1 / 2}-b / 2\right)^{-1}$, we get

$$
\limsup _{t \rightarrow-\infty} \frac{\log M_{t}^{u}(\alpha|t|)}{t}<0 .
$$

Since for bounded $M_{t}^{u}(\alpha|t|)(-\infty<t \leqq 0)$

$$
\limsup _{t \rightarrow-\infty}\left(\log M_{t}^{u}(\alpha|t|)\right) / t \geqq 0,
$$

the theorem is proved.

4. In the special case $b=0$, (4) becomes $\alpha>(c+\gamma) \gamma^{-1 / 2}$. In the following theorem we release the assumption $\gamma>0$.

THEOREM 2. Let $u(x, t)$ be a nontrivial nonnegative solution of

$$
\frac{\partial u}{\partial t}=\sum_{i, j=1}^{m} a_{i j} \frac{\partial^{2} u}{\partial x_{i} \partial x_{j}}+c u
$$

in the half space $D$ and let $\left(a_{i j}\right)$ be a positive matrix. If $u(x, t) \not \equiv A e^{c t}$, then $u(x, t)$ cannot be bounded in every cone $D_{\alpha}$.

Proof. From (19) it is clear that if $\alpha$ satisfies

$$
h(\alpha)+c<0 \text {, }
$$


then $u(x, t)$ is unbounded in $D_{\alpha}$. Here, $h(\alpha)$ is defined with respect to the function $v(|x|, t)$ obtained by symmetrization of $e^{-c t} u(T x, t)$. Since the last function is not constant, $v(|x|, t)$ is also not constant, and a simple argument based on (7) shows that $h(\alpha) \rightarrow-\infty$ as $\alpha \rightarrow \infty$. It follows that (21) is satisfied for large $\alpha$.

In the case $c=0$ we can solve (21) by using Lemmas 1 and 2. We get

Corollary. Let $u(x, t)$ be a nonconstant solution in $D$ of the equation

$$
\frac{\partial u}{\partial t}=\sum_{i, j=1}^{m} a_{i j} \frac{\partial^{2} u}{\partial x_{i} \partial x_{j}} .
$$

Suppose further that $u(x, t)$ is bounded from below. Then $u(x, t)$ is unbounded in $D_{\alpha}$ for all

$$
\alpha>\left(\lim _{t \rightarrow-\infty} \frac{\log u(0, t)}{t}\right)^{1 / 2} .
$$

\section{REFERENCES}

1. S. D. Eidelman, Estimates of solutions of parabolic systems and some of their applications, Rec. Math. (Mat. Sbornik) (N.S.) vol. 33 (1953) pp. 359-382.

2. I. I. Hirschman, A note on the heat equation, Duke Math. J. vol. 19 (1952) pp. $487-492$.

UNIVERSITY OF KANSAS 